\title{
Algunas relaciones entre el holismo de la confirmación y el holismo semántico: estudio de caso*
}

Some Relations between the Confirmation Holism and Semantic Holism:

A Case Study

Santiago Ginnobili $i^{\dagger}$

\begin{abstract}
Resumen
La tesis del holismo de la confirmación aparece por primera vez presentada de manera explícita y extensa en los escritos de Duhem. Años después, es defendida por Quine, pero no en base a la discusión de teorías científicas particulares, sino en relación con su posición semántica. Desde entonces las relaciones entre el holismo semántico y el holismo de la contrastación han sido discutidas, aunque generalmente de un modo algo despegado del análisis de teorías específicas y sus relaciones. Pretendo mostrar a partir del análisis de un caso, el de las relaciones entre la genética de poblaciones y la teoría de la selección natural, un sentido en el que el holismo de la confirmación puede ser derivado del holismo semántico.
\end{abstract}

Palabras clave: holismo de la confirmación - holismo semántico - teoría de la selección natural - genética de poblaciones

\begin{abstract}
The thesis of the confirmation holism appears for the first time explicitly and extensively in the writings of Duhem. Years later, it is defended by Quine, but not based on the discussion of particular scientific theories, but in relation to its semantic position. Since then, the relations between semantic holism and confirmation holism have been discussed, although generally somewhat detached from the analysis of specific theories and their relations. I intend to show from the analysis of a case, that of the relations between population genetics and the theory of natural selection, a sense in which the holism of confirmation can be derived from semantic holism.
\end{abstract}

Keywords: confirmation holism - semantic holism - natural selection theory - population genetics

\footnotetext{
* Recibido: 15 de diciembre de 2015. Aceptado con revisiones: 13 de febrero de 2016.

† Universidad Nacional de Quilmes (UNQ)/Universidad de Buenos Aires (UBA)/Consejo Nacional de Investigaciones Científicas y Técnicas (CONICET), Argentina. Para contactar al autor, por favor, escribir a: santi75@gmail.com.

Metatheoria 5(2)(2015): 95-106. ISSN 1853-2322. eISSN 1853-2330.

(c) Editorial de la Universidad Nacional de Tres de Febrero. Publicado en la República Argentina.
} 
"La ciencia no consiste en un montón amorfo de proposiciones aisladas, sino más bien en una totalidad orgánica de teorías interrelacionadas."

C. Ulises Moulines (1991, p. 252)

\section{Introducción}

La tesis del holismo de la confirmación aparece por primera vez presentada de manera explícita y extensa en los escritos de Duhem (1914, cap. VI). Este autor establece, a partir del análisis de la práctica científica y de la estructura de las teorías, que una experiencia nunca condena a una hipótesis aislada:

Lo único que nos enseña la experiencia es que entre todas las propuestas que sirvieron para prever este fenómeno y para constatar que no se producía hay por lo menos un error, pero dónde está el error es lo que ella no nos dice (Duhem 1914, p. 281).

Esta tesis es fundamentada por Duhem a partir, no de un análisis a priori, sino de la presentación de ejemplos extraídos de la historia de la ciencia. En estos se muestra la incidencia de teorías presupuestas por los instrumentos utilizados en la experimentación, como también el papel de la interpretación de la observación y, con una concepción de las teorías científicas sofisticada y actual, la presencia de ciertos enunciados fundamentales que no se someten directamente a la experiencia, pero esenciales para la construcción de teorías que sí se someten a la experiencia (aunque por supuesto de manera no aislada).

Así concluye:

Tratar de separar cada una de las hipótesis de la física teórica de otros supuestos sobre los cuales descansa esta ciencia, para someterla aisladamente al control de la observación, es perseguir una quimera, pues la concreción y la interpretación de cualquier experiencia de la física implica la adhesión a todo un conjunto de proposiciones teóricas (Duhem 1914, p. 303).

La incidencia del holismo de la confirmación fue 'redescubierta' tiempo después por Quine (1951). Varios autores han tratado las diferencias entre las posiciones de Duhem y de Quine (p.e. Ariew 1983, Gillies 1998, cap. 5, Needham 2000, Quine 1998, Vuillemin 1998). En el caso de Quine el holismo de la confirmación no surge del análisis de la práctica científica ni de la estructura de las teorías científicas particulares, a diferencia de Duhem, sino en relación con su posición semántica. En Dos dogmas del empirismo lo cita como un hecho en contra de que las proposiciones puedan tener de manera aislada contenido empírico.

Varios autores han discutido desde entonces la relación entre el holismo de la confirmación y el holismo del significado en la argumentación quineana (p.e. Devitt 1993, Fodor 1987, cap. 3, Fodor \& Lepore 1992, cap. 2, Harrell 1996, Putnam 1998). Pero esta discusión se ha visto disociada de su primer cariz metateórico. Son los filósofos del lenguaje o de la mente los que se han visto preocupados por argumentar a favor o en contra de ambos holismos y sus relaciones y consecuencias, por cuestiones independientes de cualquier interés por como de hecho funciona la ciencia.

Mi enfoque en este trabajo será distinto. Pretendo mostrar a partir del análisis de un caso, el de las relaciones entre la genética de poblaciones (GP en adelante) y la teoría de la selección natural (TSN en adelante), un sentido en el que el holismo de la confirmación puede ser derivado del holismo semántico. Luego pretendo mostrar que este ejemplo va a favor de la restricción del holismo semántico radical y esto tiene por consecuencia, a su vez, la restricción del holismo radical de la confirmación sostenido por Quine.

El ejemplo seleccionado resulta particularmente interesante, porque, además del valor elucidatorio intrínseco de una discusión sobre la cuestión de los vínculos existentes entre estas dos teorías, la confusión con la que es tratada esta relación tanto en libros de textos científicos, como en ciertos libros de filosofía de la biología puede ser causal o sintomática de la confusión con la que se discute la 
cuestión tanto del holismo de la confirmación como del holismo semántico. En ambos casos considero que el problema consiste en la falta de una herramienta metateórica lo suficientemente potente para el tratamiento de la estructura de las teorías. Presupondré el aparato conceptual estructuralista (Balzer, Moulines \& Sneed 1987). Esta opción sólo quedará justificada por lo fructífero que sea este trabajo en el cumplimiento de sus objetivos.

Espero que este trabajo sea visto también, en el espíritu del trabajo de Duhem, como una repatriación de la polémica al terreno de filosofía especial de la ciencia de la cual surgió. Considero que es en la filosofía especial de la ciencia en donde estas discusiones pueden desestancarse y volverse progresivas.

En la parte 2 del trabajo presentaré ciertos conceptos de la metateoría estructuralista utilizados posteriormente en la discusión. En la parte 3 presentaré el caso de análisis, el vínculo entre la genética de poblaciones y la teoría de la selección natural. En la parte 4 explicaré el sentido en el que el holismo de la confirmación se vincula con el semántico y como una restricción del holismo semántico implica una restricción del holismo de la confirmación. En la parte 5 presentaré mis conclusiones.

\section{Holismo semántico en la metateoría estructuralista}

En el estructuralismo se rechaza la distinción tradicional teórico/observacional. Esta distinción encerraría en realidad dos distinciones distintas: teórico y no-teórico, y observacional y noobservacional. De las dos distinciones sólo se conserva la primera, pero sólo nominalmente, pues la teoricidad aparece relativizada a una teoría dada. ${ }^{1}$ Como explica Moulines en Pluralidad y recursión (1991), frente al operacionalismo, que hace equivaler el significado de un término teórico a los procesos físicos que pueden asociarse a él, y frente al holismo semántico radical, que sostiene que el significado de un término teórico viene determinado por toda teoría en la que aparezca dicho término, se sostiene un holismo moderado. Habría términos que dependen semánticamente de una teoría dada $T$, los términos Tteóricos, y otros que no dependerían semánticamente de $T$, que podrían depender semánticamente de otra teoría y que servirían para contrastar T, los términos T-no-teóricos (Moulines 1991, cap. II.3). Que un término dependa semánticamente de una teoría dada $T$ quiere decir que para la determinación del concepto que expresa siempre es necesario suponer la validez de las leyes de $T$, en cuyo caso estaríamos frente a un término T-teórico. Un término T-no-teórico sería aquel que expresa un concepto para cuya determinación no siempre es necesario presuponer las leyes de $T$. Determinar un concepto, en caso de que sea cualitativo, es determinar si se aplica a un objeto particular dado, y en el caso de que sea cuantitativo, determinar el valor de la magnitud para el objeto (Díez y Moulines 1997, pp. 354-356).

Más adelante volveré sobre la incidencia de esta restricción del holismo semántico radical sobre el holismo de la confirmación e introduciré más terminología estructuralista. Por el momento me basta la introducción de estos conceptos. Es interesante destacar que el holismo semántico moderado mencionado antes, en el estructuralismo, es una tesis defendida a partir del análisis de casos particulares y no por sus consecuencias epistemológicas. Paso ahora al análisis casuístico.

\section{Estudio de casos}

Para algunos TSN se define, únicamente, a partir de diferencias en el éxito de distintos organismos en la reproducción diferencial. Si esto fuese así, el principio de Hardy-Weinberg (HW en adelante), por permitir determinar (como veremos) con bastante precisión bajo ciertos supuestos que la frecuencia génica en una población no es la esperada, permitiría cuantificar la acción de la selección natural.

\footnotetext{
${ }^{1}$ Las razones por las que se rechaza la distinción teórico/observacional por inadecuada para la tarea de reconstruir las teorías científicas y sus bases empíricas se pueden encontrar en (Balzer, Moulines \& Sneed 1987, p. 48).
} 
Incluso, podría considerarse que GP brinda una versión cuantitativa de la teoría de la selección natural cualitativa propuesta por Darwin. $^{2}$

Esta idea abonaría el papel central que para algunos tiene HW en la teoría de la evolución en general. Esta sería, según ciertos autores, la ley fundamental, no sólo de la genética de poblaciones sino además de la teoría evolutiva. ${ }^{3}$ Este es el caso de Ruse quien otorga a GP capacidad de unificar a toda la biología evolutiva (Ruse 1973, cap. 4). Esto también parece estar presupuesto en autores que pretenden haber reconstruido la teoría evolutiva ofreciendo sólo una reconstrucción de GP (p.e. Barbadilla 1990, Lloyd 1994, Thompson 1989).

GP y TSN son dos teorías distintas, aunque vinculadas, alguno de cuyos vínculos, que pueden ser caracterizados como semánticos, pretendo mostrar. Un corolario del análisis que sigue consistirá en que GP no puede ser la teoría fundamental de la teoría de la evolución. Pues, existe al menos una teoría, TSN, que no puede ser incluida bajo GP en ningún sentido.

\subsection{La teoría de la selección natural}

Comenzaré exponiendo los conceptos fundamentales de la teoría de la selección natural y lo que se pretende explicar con ésta. Esto permitirá, por un lado, establecer cierto vocabulario para poder discutir cuestiones subsiguientes sin ambigüedad y permitirá establecer con claridad la diferencia y los vínculos con GP. Aunque, curiosamente, TSN es mucho más simple de comprender que GP, me veré forzado a tratar la cuestión de los conceptos fundamentales de TSN con mayor profundidad y extensión. La razón por la que tales teorías se confunden tiene que ver con una mala comprensión de la estructura de TSN y no con una mala comprensión de GP. Extraeré los conceptos fundamentales de TSN a partir de un caso de aplicación del mismo Darwin. La estructura de TSN que propongo es mucho más convincente al abstraerla de muchos casos, pero esto excede los límites de este trabajo. ${ }^{4}$

Como es bien sabido, lo que Darwin quiere explicar con TSN, es cierta adecuación o ajuste de los organismos al medio ambiente.

Por ejemplo:

La jirafa, con su gran estatura, sus muy largos cuello, patas delanteras, cabeza y lengua, tiene su estructura bellamente adaptada para comer en las ramas más altas de los árboles. Puede por eso obtener comida fuera del alcance de otros ungulados que habitan el mismo lugar; y esto debe ser una gran ventaja durante períodos de escasez (Darwin 1872, p. 161).

La forma en que Darwin explica la fijación de este rasgo en la población de jirafas es la siguiente:

En la naturaleza, en el origen de la jirafa, los individuos que comiesen más alto y que pudiesen durante los periodos de escasez alcanzar, aunque sea una pulgada o dos por sobre los otros, serían frecuentemente preservados [...]. El que los individuos de la misma especie muchas veces difieren un poco en la longitud relativa de todas sus partes, puede comprobarse en muchas obras de historia natural en las que se dan medidas cuidadosas. Estas pequeñas diferencias en las proporciones, debidas a las leyes de crecimiento o variación, no tienen la menor importancia ni utilidad en la mayor parte de las especies. Pero en el origen de la jirafa debe hacer sido diferente, considerando sus probables hábitos de vida; pues aquellos individuos que tuviesen alguna parte o varias partes de su cuerpo un poco más alargadas de lo corriente, hubieron en general de sobrevivir. Se habrán cruzado y dejado descendencia que habrán heredado las mismas peculiaridades corpóreas, o la tendencia a variar de nuevo en la misma manera, mientras que los individuos menos favorecidos en los mismos aspectos, habrán sido más propensos a perecer (Darwin 1872, p. 161, itálicas mías).

\footnotetext{
${ }^{2}$ La utilización del término 'teoría' en este artículo se referirá en todos los casos a lo que en la metateoría estructuralista es llamado 'red teórica’ (Balzer, Moulines \& Sneed 1987, cap. IV). Presupondré en este artículo que TSN y GP son redes teóricas. Argumentaré, por supuesto, que se trata de redes teóricas distintas.

${ }^{3}$ Si bien sólo intentaré mostrar que no puede tratarse de la ley fundamental de la teoría de la evolución, tampoco es adecuado considerar HW como la ley fundamental de GP. Para un tratamiento de esta cuestión ver (Lorenzano 2008).

${ }^{4}$ Para un análisis más extenso de la estructura de TSN ver (Ginnobili 2006a, b, 2007a, b, 2009, 2010a, b, 2011).
} 
Si consideramos, con los estructuralistas, que la ley fundamental de TSN es aquél enunciado en el que aparecen (frecuentemente todos) los conceptos fundamentales de TSN relacionados (Balzer, Moulines \& Sneed 1987, p. 19, Lorenzano 2005), podemos extraer una instanciación de TSN a partir de la explicación citada:

Las jirafas con cuello, patas delanteras, cabeza y lengua de mayor longitud son más efectivas al alimentarse de las ramas más altas de los árboles, mejorando su supervivencia y mejorando, en consecuencia, su éxito reproductivo diferencial.

Nada de este enunciado parece superfluo. Si quitamos alguna de sus partes la explicación brindada con él quedaría trunca. Si se quita la función dada al rasgo por el organismo, la de alcanzar las ramas altas de los árboles, no sabríamos por qué tal rasgo podría mejorar la supervivencia. Podría mejorarla permitiendo asustar a posibles predadores, lo que constituiría una explicación alternativa y competidora de la ofrecida por Darwin. Sí quitamos la mejora en la supervivencia quedaría indeterminada la relación que hay entre el rasgo y el éxito reproductivo. No sabríamos si se trata de un caso de selección sexual, por ejemplo, siendo el rasgo y su capacidad atractivos en algún sentido a las hembras de la misma especie. ${ }^{5}$

Por abstracción de este enunciado, nos vamos acercando a lo que considero que es la ley fundamental de TSN:

Los individuos con rasgos que cumplen con mayor efectividad cierta función, mejoran su supervivencia mejorando su éxito en la reproducción diferencial.

La ley fundamental de TSN tendría al menos tres componentes:

- El rasgo que cumple de manera más adecuada una función

- El éxito reproductivo diferencial, que tiene que ver con alguna medida de la cantidad de descendencia fértil dejada por los organismos.

- La conexión entre el rasgo adecuado y el éxito reproductivo, que llamaré "aptitud". En este caso se trata de una mejora en la supervivencia, pero en otros casos puede tratarse de una mejora en la capacidad de conseguir parejas -selección sexual-, de una mejora en la fecundidad -p.e. plantas que mejoran su éxito reproductivo atrayendo a insectos con sus flores-, etc. (Ginnobili 2006b, 2010a, b, 2011).

No pretendo llevar esta elucidación más allá de lo necesario para el punto defendido en este trabajo. En particular no trataré aquí el estatus del concepto que he llamado "aptitud". Basta con señalar que se trata de un concepto abstracto que recibe diferentes interpretaciones en las leyes especiales de TSN, permitiendo realizar aplicaciones diversas. Discutiré, porque es fundamental en este trabajo, el estatus de teoricidad de los otros dos conceptos.

Normalmente los conceptos teóricos de una teoría dada permiten explicar hechos descriptos o interpretados a partir de los conceptos no-teóricos de esa teoría. Así, los conceptos de fuerza y masa en la mecánica clásica permiten explicar las aceleraciones de las partículas. Fuerza y masa son conceptos teóricos en la mecánica clásica, puesto que no es posible aplicarlos independientemente de esta teoría, mientras que aceleración es un concepto no-teórico en la mecánica clásica, puesto que sí es posible aplicarlo independientemente de esta teoría.

Siendo así, que un organismo posea un rasgo que permita realizar una función con cierta efectividad debiera poder ser establecido con independencia de TSN. Y así es, efectivamente, pues esto mismo es lo que los teólogos naturales querían explicar apelando a un diseñador inteligente (Blanco 2008). Que las jirafas utilizan el cuello para alcanzar las ramas altas de los árboles puede ser determinado por el estudio del comportamiento de las jirafas sin necesidad de la postulación de

\footnotetext{
5 Supongo aquí que la selección sexual es un caso de la natural. Para esta cuestión se puede consultar (Endler 1986, Ginnobili 2006b, por aparecer).
} 
ningún mecanismo evolutivo y con independencia del origen histórico filogenético del rasgo. Esto aparece en la pregunta que se quiere contestar con TSN, tal como vimos anteriormente.

También puede ser determinado con independencia de TSN el éxito reproductivo de los organismos. Sea como fuere que se realice esta medición, evidentemente será de manera independiente de TSN, puesto que surgirá mediata o inmediatamente del conteo de descendencia fértil o viable de estos organismos.

Así, TSN tiene al menos dos conceptos no-teóricos: adecuación y éxito reproductivo. Siendo así, con TSN es posible dar dos tipos de explicaciones diferentes:

- Explicación del surgimiento de la adecuación. Para esto fue propuesta TSN por Darwin, para explicar el surgimiento de adecuaciones al ambiente sin necesidad de postular ningún tipo de diseñador inteligente. Para esto apela a los conceptos de aptitud y de éxito reproductivo.

- Explicación de las diferencias en el éxito en la reproducción diferencial: Es posible también explicar por qué determinados individuos tienen más éxito que otros apelando a los conceptos de aptitud y de adecuación ${ }^{6}$.

A Darwin no le interesaba particularmente este último tipo de explicación, pues no tenía forma de determinar con claridad el éxito reproductivo de los organismos vivos. Esta situación, como veremos, cambio abruptamente con el tiempo. Con el descubrimiento de HW y con el decaimiento de las explicaciones que incluían diseñadores inteligentes, al menos dentro del seno de la biología, esta es la función explicativa más importante de TSN. Pues es posible ahora establecer cuantitativamente GP con cierta precisión la frecuencia esperada de rasgos en una población si se cumplen ciertos supuestos. Si la frecuencia no es la esperada, una de las posibles explicaciones es, justamente, que existe una presión selectiva a favor de los organismos que poseen ciertos rasgos. Veremos esto con mayor detenimiento.

\subsection{Genética de poblaciones}

Mi intención es ahora dar una breve presentación de GP, en particular, de HW, funcional a los fines de este trabajo. En este caso no la reconstruiré, sino que seguiré simplificadamente las presentaciones habituales de los libros de texto de biología evolutiva.

Hardy y Weinberg, de modo independiente mostraron, a comienzos del siglo XX, cuál debiera ser la frecuencia genética esperada en una población dada que cumple con las siguientes condiciones (Futuyma 1986, pp. 83-87):

1. El tamaño de la población es infinito -en una población finita la frecuencia de los alelos puede fluctuar de generación en generación debido a eventos azarosos (deriva génica)-.

2. Los individuos se aparean unos con otros azarosamente.

3. No ocurren mutaciones.

4. No hay migraciones de ningún tipo.

5. Los alelos son igualmente competentes para hacer copias de sí mismos, entrando en el pool genético de los gametos.

En una población ideal en la que se cumplieran estos supuestos Hardy y Weinberg mostraron matemáticamente para un único gen que posee dos alelos ( $\mathrm{A}$ y $a$ ), que las frecuencias relativas de estos alelos no cambiaran de una generación a otra. El reservorio génico con respecto a estos alelos estará en el estado de equilibrio expresado en la ecuación: $\mathrm{p}^{2}+2 \mathrm{pq}+\mathrm{q}^{2}=1$. En donde $\mathrm{p}^{2}$ permitirá establecer la frecuencia de los genotipos AA -los homocigotos dominantes-, 2pq la frecuencia de los fenotipos Aalos heterocigotos- y $\mathrm{q}^{2}$ la frecuencia de los genotipos a - -los homocigotos recesivos.

\footnotetext{
${ }^{6}$ Estoy tratando la cuestión de los diferentes explananda de TSN de un modo simplificado. Para un tratamiento más detallado de esta cuestión se puede apelar a otros textos (Ginnobili 2010a, 2011). En esas ocasiones, mostré que en realidad es mejor separar la teoría de la selección natural histórica, que da cuenta del origen de la adecuación, de la ahistórica que permite explicar el éxito reproductivo. Para los fines de este artículo, estas diferencias pueden dejarse de lado.
} 
Si en una población dada la frecuencia génica para un gen con dos alelos no es la esperada, entonces podemos inferir que alguno de los supuestos señalados no está siendo cumplido. Supongamos un caso en el que una población determinada (1) es lo suficientemente grande como para que los errores muestrales de la deriva génica sean desestimables, (2) está lo suficientemente aislada como para que el efecto de las migraciones sea desestimable, (3) no hay razones para pensar que el apareamiento no sea azaroso (por ejemplo, no hay autopolinización) y (4) el efecto de la mutación es desestimable (normalmente este es el caso puesto que las tasas de mutación son bajas). ¿Cómo explicamos que la frecuencia para esos alelos no es la esperada? Podemos apelar a TSN. Probablemente alguno de los alelos esté sufriendo una presión selectiva. Como vimos Darwin había propuesto TSN para dar una explicación histórica del origen de la adecuación. Hoy, TSN también tiene la función de aplicarse justamente en este caso. Uno de los alelos está teniendo más éxito que el otro en reproducirse porque incrementa la aptitud de los organismos que lo portan.

\subsection{Vínculos interteóricos}

Si esta presentación es correcta, entonces queda claro que TSN y GP son teorías distintas. Y que en el caso presentado GP permitió determinar uno de los conceptos de TSN, el de éxito reproductivo, que como veíamos es un concepto TSN no-teórico. GP funcionaría en casos como el relatado en la 'base empírica' de TSN.

Sin embargo, los libros de texto de biología evolutiva suelen oscilar a la hora de conceder un estatus independiente de GP a TSN. Así, Futuyma, quien concede en su reconocido libro (Futuyma 1986) un papel explicativo central a TSN -el capítulo 6 se llama "Efectos de la selección natural en las frecuencias génicas" - afirma: "Si los alelos difieren en sus tasas de reemplazo, sus frecuencias pueden cambiar. Este fenómeno es llamado "selección”" (Futuyma 1986, p. 87). Es decir, la selección natural parece identificarse en esta cita con la mera diferencia en el éxito reproductivo. Esto no sólo ocurre en libros de texto científicos. Gayon, reconocido historiador de la ciencia, afirma: "La selección natural no es más un principio fundamental sino un parámetro que mide una de las tantas fuerzas de cambio" (Gayon 1998, p. 321), en referencia a los cambios producidos por el descubrimiento de GP.

¿Es posible considerar que GP brindó una versión cuantificada de lo que Darwin había presentado de manera cualitativa?

Del mismo modo que en un mundo newtoniano podría inferirse a partir de que una partícula se ha acelerado, que una fuerza -o una interacción de fuerzas- ha actuado, en un mundo en el que migración, deriva génica, mutación, apareamiento selectivo y selección natural fuesen las únicas fuerzas que actuaran sobre las frecuencias génicas de una población, uno podría inferir que la selección natural ha actuado a partir de que la frecuencia génica en una población no es la esperada y de la constatación de que ninguno de las otros factores tomados en cuenta en HW han intervenido. Pero señalar que hay razones para creer que la selección natural ha actuado no es equivalente a aplicar TSN, del mismo modo que en este supuesto mundo newtoniano, señalar que una fuerza o una combinación de fuerzas es la responsable del cambio de aceleración, no es aplicar la mecánica clásica de partículas. Una aplicación de la mecánica clásica de partículas implica determinar el número y la naturaleza de las fuerzas en acción. De igual modo, aplicar TSN implica encontrar el rasgo que está siendo utilizado de manera más efectiva y la conexión entre tal rasgo y el éxito reproductivo, que yo he llamado "aptitud". Así como en el ejemplo newtoniano puede haber una combinación de fuerzas de diferente naturaleza actuando sobre una partícula, puede ocurrir lo mismo en la aplicación de TSN. La presión selectiva podría ser producida por una combinación entre, por ejemplo, supervivencia y capacidad de atracción de parejas de apareamiento. En conclusión, la mera determinación del éxito reproductivo, no es la aplicación de TSN.

Insisto, dada la estructura genética de una población, la mera predominancia de un rasgo fenotípico por sobre otro no permite inferir que los organismos que portan dicho rasgo están teniendo éxito en la competencia por dejar descendencia por sobre otros organismos con otros rasgos fenotípicos. Puede tratarse de un rasgo determinado por un genotipo dominante, por ejemplo. Por lo 
tanto, lo que permite HW en GP es una sofisticación y mejora de la medición del éxito reproductivo. Pero esto no es de ningún modo la medida de la acción de TSN. ${ }^{7}$

Este largo rodeo nos permitió establecer que TSN y CP son dos teorías diferentes, ninguna de las cuales es especialización de la otra. Contra los que pretendían, como veíamos, conceder un estatus privilegiado a GP en la teoría evolutiva, existe al menos una teoría independiente y distinta de GP en la teoría evolutiva: TSN.

Podemos pasar ahora a la cuestión central de este artículo, evidentemente, como hemos visto en esta larga digresión, hay vínculos entre ambas teorías. El paso siguiente es analizar al menos alguno de ellos.

En el marco conceptual estructuralista se propone una clasificación de vínculos que dos teorías pueden tener (Balzer, Moulines \& Sneed 1987, cap. VI, Moulines 1991, cap. III.3). Me enfocaré en los vinculos interteóricos determinantes [presupposition links] (Balzer, Moulines \& Sneed 1987, pp. 57-62). La naturaleza de este tipo de vínculos es semántica. Son los vínculos por los cuales un concepto de una teoría es utilizado en otra, es decir, cuando en una teoría dada aparece un concepto que puede ser determinado utilizando otra teoría. Se llama a este tipo de vínculos "determinantes" para distinguirlos de los vínculos implicativos que son de naturaleza global, que conectan leyes y no conceptos. Tal vez se podría caracterizar a otros vínculos como semánticos, pero me enfocaré sólo en estos.

Estos vínculos, en tanto transmiten información de una teoría a otra, evidentemente abonan cierta tesis holista del significado. Veíamos sin embargo en la sección 1 de este trabajo el sentido en el cual habría que restringir la tesis del holismo radical, según la cual el significado de un concepto está determinado por todas las teorías en las que aparece. Un concepto puede aparecer en una teoría como no-teórico en esa teoría, en cuyo caso su significado no depende semánticamente de esa teoría.

Esta cuestión puede ser planteada también del siguiente modo. Los vínculos determinantes no son simétricos. Puede haber un traspaso de información de la teoría A a la teoría B sin que haya traspaso de información de la teoría B a la A. Así, una teoría depende semánticamente de las teorías desde las cuales se determinan sus conceptos no-teóricos. Pero estas teorías no dependen semánticamente, o al menos no necesariamente, de aquella.

Volvamos sobre nuestro caso de estudio. Concediendo lo máximo que se puede conceder a aquellos que sostienen un lugar esencial de GP en la teoría evolutiva, si lo que he defendido hasta aquí es adecuado: asumamos que GP es necesaria para la determinación del éxito reproductivo de los individuos, y por lo tanto, necesariamente presupuesta en toda aplicación actual de $\mathrm{TSN}^{8}$. En este sentido habría un vínculo determinante que conecta el concepto éxito reproductivo de TSN con un el concepto de éxito reproductivo en GP (lo que suele llamarse en GP 'éxito reproductivo' o 'aptitud' y que no habría que confundir con el concepto de aptitud de TSN). TSN dependería semánticamente de GP, pero no ocurriría lo inverso.

\section{Restricción del holismo de la confirmación}

Las predicciones no son hechas a partir de hipótesis particulares sino suponiendo entramados teóricos completos y complejos. De ocurrir o no la predicción, lo confirmado o refutado es el entramado teórico de manera general. En el caso de la refutación esto es particularmente grave porque no queda

\footnotetext{
7 Glymour (2006) ataca también esta idea, aunque por medios diferentes. Pero no puedo más que concordar con sus conclusiones cuando afirma: "La genética de poblaciones nos ha dado un conjunto de herramientas útiles para representar los cambios de frecuencia en las poblaciones naturales y para describir procesos de selección. Estos usos son importantes, incluso esenciales, en la mayor parte de la biología evolutiva y poblacional. Pero, mayormente por un accidente histórico, muchos biólogos y casi todos los filósofos han sobrestimado a la genética de poblaciones, en tanto, implícita o explícitamente, la genética de poblaciones es considerada como proveyendo el corazón formal de la maquinaria para describir procesos selectivos" (Glymour 2006, pp. 388).

8 Si este fuera el caso, tal vez habría que conceder que con la síntesis TSN sufrió un cambio esencial, puesto que para el estructuralismo los vínculos determinantes son esenciales a las teorías. En este sentido se podría decir que la teoría de la selección natural de Darwin y la actual son diferentes. Pero no afirmo que sea el caso. Esto implicaría sostener que el éxito reproductivo sólo puede ser determinado en casos de rasgos determinados genéticamente, y por genes en el sentido clásico. Esto podría implicar una restricción en el campo de aplicación de TSN. No podría aplicarse, por ejemplo, a rasgos que de transmisión cultural. No trataré estas cuestiones en este trabajo.
} 
claro qué parte de este entramado es la responsable del error (si es que se puede hablar de que una parte lo sea). ${ }^{9}$ Esto es lo que ha sido llamado 'holismo de la confirmación'.

La visión del holismo de la confirmación que presenta Quine en Dos dogmas del empirismo afirma que el entramado de teorías que se ve confirmado o refutado incluye a todo el conocimiento humano en ciencias formales y fácticas (Quine 1951, pp. 42-46). ${ }^{10}$ Creo que es sencillo mostrar en qué sentido esta idea es exagerada.

Como hemos visto, la investigación metateórica muestra que el holismo semántico radical es inadecuado para comprender las relaciones entre los conceptos de las teorías científicas. Considero que lo mismo puede ser afirmado al respecto del holismo de la confirmación radical presentado por Quine. Esto no es una mera analogía, dado que el holismo semántico es una de las causas del holismo de la confirmación, como ahora mostraré, la restricción en el primero restringe automáticamente al segundo.

Así, en una contrastación no sólo se presuponen teorías, por ejemplo, al respecto de los instrumentos utilizados, sino, además, teorías con las que la teoría a contrastar tiene vínculos interteóricos determinantes. Este hecho no había sido tomado en cuenta en las discusiones habituales porque tales vínculos han pasado inadvertidos en las reconstrucciones tradicionales (Balzer, Moulines \& Sneed 1987, p. 59). Si una teoría H' es presupuesta en la determinación de un concepto no-teórico de la teoría $\mathrm{H}$, entonces, dentro de todo lo que se presupone para contrastar $\mathrm{H}$ estará incluida la teoría $\mathrm{H}^{\prime}$. Así, el vínculo semántico entre $\mathrm{H}$ y H' genera una vinculación entre ambas teorías a la hora de la contrastación. En el caso examinado, si GP es presupuesta en la aplicación de TSN entonces formara parte del entramado teórico a contrastar en las aplicaciones de TSN.

Pero, recordemos que los vínculos determinantes no son simétricos. Esto restringe el holismo de la confirmación. Al contrastar GP, se supondrán todas las teorías presupuestas por GP. Pero entre ellas no se encontrará TSN. Al menos no tomando en cuenta únicamente el vínculo analizado.

Como ya se ha señalado anteriormente, esto presupone que GP es necesaria para la determinación del éxito reproductivo. Si consideramos que esto no es cierto, entonces, podemos restringir todavía más el holismo. GP sólo estará en juego en aquellas aplicaciones de TSN en las que se haya utilizado.

Por supuesto, determinar el entramado de relaciones entre las teorías que conforman lo que los biólogos evolutivos suelen llamar 'teoría evolutiva', es una tarea ardua que apenas se ha comenzado a realizar. Cual sea el conocimiento presupuesto en aplicaciones particulares, y cual sea, en consecuencia, el alcance del holismo de la confirmación, será un resultado de esta investigación metateórica. Lo único que quiero señalar es, por un lado, que, si se deriva el holismo de la confirmación del holismo semántico, el debilitamiento del holismo semántico radical podría ser síntoma, y creo que lo es, de un debilitamiento correspondiente en el holismo de la confirmación. Por otro lado, que no siempre se presupone el mismo cuerpo de conocimiento en cada aplicación de una teoría. GP es presupuesta por TSN sólo cuando es utilizada para determinar el éxito reproductivo diferencial. Finalmente, y tal vez principalmente, espero que haya quedado claro que no basta con la afirmación de que existen vínculos entre las teorías que conforman la biología evolutiva para defender una tesis al respecto del alcance del holismo de la confirmación. Es posible analizar estos vínculos, y por lo tanto, es necesario hacerlo para asumir una posición en esta cuestión.

\footnotetext{
9 Si estamos utilizando una metateoría como la estructuralista, es necesario cualificar el significado de conceptos como refutado o confirmado, puesto que son utilizados y elucidados normalmente, desde metateorías enunciativistas clásicas. Utilizaré estos conceptos de manera presistemática para señalar sólo el éxito o el fracaso en la aplicación de una teoría a un caso pretendido. El mismo Duhem tenía una posición compleja respecto de la posibilidad de falsar las teorías. Tanto en el estructuralismo como en Duhem la razón por la cual la teoría no es falsable en un sentido clásico es independiente de la cuestión del holismo de la confirmación. Es posible, de todos modos, relacionar estas dos cuestiones (Gillies 1998, cap. 10). Para el caso de la falsabilidad de TSN puede acudirse a Ginnobili (2007b).

${ }^{10}$ Ciertamente este no parece ser el caso en los escritos de Duhem (Gillies 1998, pp. 111-112, Lorenzano 2008).
} 


\section{Conclusiones}

He intentado mostrar, ilustrándolo con un ejemplo, el sentido en que el holismo semántico se relaciona en ciertos casos con el holismo de la confirmación. También he intentado mostrar que el hecho de que el análisis de teorías científicas particulares muestre que es necesario restringir el holismo semántico radical, implica la restricción del holismo de la confirmación radical.

Alguien podría objetar al presente trabajo el largo camino realizado a través del caso en cuestión. El autor podría haber tomado un atajo: pues bastaba exponer los conceptos estructuralistas mostrando las consecuencias que esto acarrea para la cuestión del holismo de la confirmación citando algún texto de referencia. El interesado podría acceder allí a decenas de casos en apoyo de la metateoría en cuestión. Pero el rodeo a través del ejemplo fue premeditado. Existe una buena razón por la que elegí el camino más extenso. Me refiero a que, además de servir como ilustración de los temas discutidos, el caso permite contrastar esta estrategia con la vaguedad con que a menudo ciertas cuestiones son tratadas en la filosofía analítica y en las filosofías especiales de la ciencia. En ambos casos esta vaguedad tiene origen, creo, en el desprecio que se tiene por herramientas metateóricas y marcos generales para el estudio de la estructura de las teorías científicas producidas en el ámbito de la filosofía de la ciencia. Previo a un análisis metateórico ni siquiera es posible determinar si GP y TSN son teorías distintas y establecer los vínculos que las unen. Si la discusión acerca del holismo de la confirmación utiliza herramientas igual de imprecisas para hablar de la ciencia, cualquier posición es defendible. La caída de la concepción de teoría científica propuesta por los empiristas lógicos -provocadas en gran medida por sus propias investigaciones (ver p.e. Hempel 1970)- parece haber provocado la caída de las metas reconstructivas que se proponían. Dentro del programa estructuralista, que yo he venido utilizando de manera informal en este trabajo, se ha conservado este espíritu con numerosísimos resultados exitosos. Espero que uno de estos sea el análisis presente. Independientemente de lo correcto de mis afirmaciones particulares, considero que las críticas más fructíferas a este trabajo supondrán al estructuralismo o una herramienta metateórica de la misma sofisticación. Tanto Quine (con respecto al holismo de la confirmación) como Ruse (con respecto al papel unificador de GP) hablan de la estructura de las teorías de manera vaga. Si bien ambos textos llevan a la fecha varios años desde su aparición, la actitud general al respecto de esta cuestión no ha cambiado mucho. Creo que la aplicación del estructuralismo ha echado luz parcial, al menos, sobre las cuestiones que ambos discuten.

Finalmente, creo que tanto la cuestión del holismo de la confirmación, como del holismo semántico (al menos cuando se refiere a la cuestión del significado de los conceptos del lenguaje científico especializado) pertenece al ámbito de la filosofía de la ciencia especial. Sólo la reconstrucción de las teorías científicas particulares y de sus vínculos puede mostrar el entramado del conocimiento científico que es puesto a prueba en los casos particulares de contrastación. La discusión de estas cuestiones a priori e independiente de lo aprendido en el análisis de las teorías y la práctica científica, no tiene otro destino que el escolasticismo y el estancamiento.

Bibliografía

Ariew, R. (1983), “The Duhem Thesis”, British Journal for the Philosophy of Science 35: 313-325.

Balzer, W., Moulines, C. U. y J. D. Sneed (1987), An Architectonic for Science - The Structuralist Program, Dordrecht: Reidel. (Versión castellana de Pablo Lorenzano: Una arquitectónica para la ciencia. El programa estructuralista, Bernal: Universidad Nacional de Quilmes, 2012.)

Barbadilla, A. (1990), "La estructura de la teoría de la selección natural”, en Ruiz, A. y M. Santos (eds.), Temas actuales de Biología Evolutiva, Barcelona: UAB, pp. 163-191.

Blanco, D. (2008), "La naturaleza de las adaptaciones en la teología natural británica: análisis historiográfico y consecuencias metateóricas”, Ludus Vitalis 16(30): 3-26. 
Darwin, C. (1872), The Origin of Species, $6^{\mathrm{a}}$ ed., London: John Murray.

Devitt, M. (1993), "A Critique of the Case for Semantic Holism", Philosophical Perspectives 7: 281-306.

Duhem, P. (1914), La théorie physique: son objet et sa structure (deuxième édition), Paris: Marcel Rivière \& Cte, éditeurs.

Endler, J. A. (1986), Natural Selection in the Wild, Princeton, New Jersey: Princeton University Press.

Fodor, J. A. (1987), Psychosemantics, Cambridge: MIT Press.

Fodor, J. A. y E. Lepore (1992), Holism: A Shopper's Guide, Oxford: Blackwell.

Futuyma, D. J. (1986), Evolutionary Biology, 2ª ed., Sunderland, MA: Sinauer Associates, Inc.

Gayon, J. (1998), Darwinism's Struggle for Survival: Heredity and the Hypothesis of Natural Selection, Cambridge: Cambridge University Press.

Gillies, D. (1998), Philosophy of Science in the Twentieth Century - Four Central Themes. Oxford/Cambridge: Blackwell.

Ginnobili, S. (2006a), "La selección natural como conjunto de hechos e inferencias", en Ahumada, J., Pantalone, M. y V. Rodríguez (eds.), Epistemología e Historia de la Ciencia, Córdoba: Universidad Nacional de Córdoba, pp. 266275.

Ginnobili, S. (2006b), La teoría de la selección natural darwiniana, Tesis de licenciatura, Buenos Aires: Universidad de Buenos Aires.

Ginnobili, S. (2007a), "Darwinismo universal de dominio de aplicación restringido", en en Martins, L. A.C. P., Prestes, M. E. B., Stefano, W. y R. de A. Martins (eds.), Filosofia e história da biologia 2, São Paulo: Fundo Mackenzie de Pesquisa (MackPesquisa), pp. 427-443.

Ginnobili, S. (2007b), "Hay lo que queda. Sobre la presunta tautologicidad de la teoría de la selección natural", Análisis Filosófico 27(1): 75-89.

Ginnobili, S. (2009), "El poder unificador de la teoría de la selección natural”, en Barboza, M. C., Avila, J. D., Píccoli, C. y J. Cornaglia Fernández (eds.), 150 años después... La vigencia de la teoría evolucionista de Charles Darwin, Rosario: Universidad Nacional de Rosario, pp. 141-154.

Ginnobili, S. (2010a), "La teoría de la selección natural darwiniana”, Theoria 25(1): 37-58.

Ginnobili, S. (2010b), "La teoría de la selección natural darwiniana y la genética de poblaciones", Endoxa 24: 169-184.

Ginnobili, S. (2011), La estructura de la teoría de la selección natural. Elucidación de sus conceptos fundamentales, reconstrucción de su estructura y consecuencias del análisis sobre algunas discusiones metateóricas a su alrededor, Tesis doctoral, Buenos Aires: Universidad de Buenos Aires.

Ginnobili, S. (por aparecer), "Selección artificial, selección sexual, selección natural”, Metatheoria.

Glymour, B. (2006), "Wayward Modeling: Population Genetics and Natural Selection”, Philosophy of Science 73(4): 369389.

Harrell, M. (1996), “Confirmation Holism and Semantic Holism”, Synthese 109: 63-101.

Hempel, C. G. (1970), “On the 'Standard Conception' of Scientific Theories”, en Radner, M. y S. Winokur (eds.), Minnesota Studies in the Philosophy of Science, Minneapolis: University of Minnesota Press, pp. 142-163.

Lorenzano, P. (2005), “Ejemplares, modelos y principios en la genética clásica”, Scientiae Studia 3(2): 185-203.

Lorenzano, P. (2008), "Bas C. van Fraassen y la ley de Hardy-Weinberg: una discusion y desarrollo de su diagnóstico", Principia 12(2): 121-154.

Lloyd, E. A. (1994), The Structure and Confirmation of Evolutionary Theory, New Jersey: Princeton University Press.

Moulines, U. (1991), Pluralidad y recursión, Madrid: Alianza.

Needham, P. (2000), “Duhem and Quine”, Dialectica 54(2): 109-132.

Putnam, H. (1998), "Meaning Holism”, en Hahn, L. E. y P. A. Schilpp (eds.), The Philosophy of W. V. Quine, ed. extendida, Chicago/La Salle: Open Court, pp. 405-426.

Quine, W. V. O. (1951), "Main Trends in Recent Philosophy: Two Dogmas of Empiricism”, The Philosophical Review 60(1): 20-43 
106 | Santiago Ginnobili

Quine, W. V. O. (1998), “Reply to Jules Vuillemin”, en Hahn, L. E. y P. A. Schilpp (eds.), The Philosophy of W. V. Quine, ed. extendida, Chicago/La Salle: Open Court, pp. 619-622.

Ruse, M. (1973), The Philosophy of Biology, London: Hutchinson \& Co.

Thompson, P. (1989), The Structure of Biological Theories, New York: State University of New York Press.

Vuillemin, J. (1998), “On Duhem's and Quine's Theses”, en Hahn, L. E. y P. A. Schilpp (eds.), The Philosophy of W. V. Quine, ed. extendida, Chicago/La Salle: Open Court, pp. 595-618. 